\title{
AGILE Highlights after Six Years in Orbit
}

\author{
Carlotta Pittori ${ }^{1,2}$ on behalf of the AGILE Collaboration \\ ${ }^{1}$ ASI Science Data Center (ASDC), via del Politecnico snc, 00133 Roma, Italy. \\ ${ }^{2}$ INAF-OAR, Via Frascati 33, I00040 Monte Porzio Catone (RM), Italy. \\ Corresponding author: carlotta.pittori@asdc.asi.it
}

\begin{abstract}
AGILE is an ASI space mission in collaboration with INAF, INFN and CIFS, dedicated to the observation of the gammaray Universe in the $30 \mathrm{MeV}-50 \mathrm{GeV}$ energy range, with simultaneous X-ray imaging capability in the $18-60 \mathrm{keV}$ band. The AGILE satellite was launched on April 23rd, 2007, and produced several important scientific results, among which the unexpected discovery of strong flares from the Crab Nebula. This discovery won to the AGILE PI and the AGILE Team the Bruno Rossi Prize for 2012 by the High Energy Astrophysics division of the American Astronomical Society. Thanks to its sky monitoring capability and fast ground segment alert system, AGILE detected many Galactic and extragalactic sources: among other results AGILE discovered gamma-ray emission from the microquasar Cygnus X-3, detected many bright blazars, discovered several new gamma-ray pulsars, and discovered emission up to $100 \mathrm{MeV}$ from Terrestrial Gamma-Ray Flashes. We present an overview of the main AGILE Data Center activities and the AGILE scientific highlights after 6 years of operations.
\end{abstract}

Keywords: Gamma-rays: observations - catalogs.

\section{Introduction}

AGILE (Astro-rivelatore Gamma a Immagini LEggero) (Tavani et al., 2009a) is a gamma-ray astrophysics mission of the Italian Space Agency (ASI), with INAF, INFN and CIFS participation which has been in orbit since 23 April 2007.

The AGILE payload is composed of a pairproduction Gamma Ray Imager (GRID) sensitive in the energy range $30 \mathrm{MeV}-50 \mathrm{GeV}$ (Barbiellini et al., 2001; Prest et al., 2003), an X-ray Imager (SuperAGILE) sensitive in the energy range 18-60 keV (Feroci et al., 2007), and a Mini-Calorimeter (MCAL) sensitive to gamma-rays and charged particles with energies between $300 \mathrm{keV}$ and $100 \mathrm{MeV}$ (Labanti et al., 2009). The instrument, weighting only $\sim 100 \mathrm{~kg}$, is the most compact ever operational for high-energy astrophysics (approximately a cube of about $60 \mathrm{~cm}$ size) with excellent detection and imaging capability. AGILE is characterized by a very large field of view $(\sim 3 \mathrm{sr})$, a good angular resolution, 0.1 - 0.2 degrees in gamma rays and 1-2 arcminutes in $\mathrm{X}$ rays, and a small dead time (100 $\mu \mathrm{s})$, which make it a very good instrument to study persistent and transient gamma-ray sources even on very short timescales.

Recent calibration results of the AGILE-GRID using in-flight data and Monte Carlo simulations producing instrument response functions have been presented in Chen et al. (2013).

\section{The Ground Segment and the AGILE Data Center}

The AGILE Data Center (ADC) is part of the ASI Science Data Center (ASDC), previously located in the ESA establishment of ESRIN in Frascati, and recently moved within the ASI Headquarters, Rome (Italy). Satellite data are routinely collected every $\sim 96 \mathrm{~min}-$ utes by the ASI ground station in Malindi (Kenya), then quickly sent to the Satellite Operations Centre in Telespazio, Fucino, and then transferred, preprocessed, analyzed, and stored at the ADC. ADC is in charge of all the scientific oriented activities related to the analysis, archiving and distribution of AGILE data. From July 2007 July to October 2009 ( 2.5 years) AGILE was operated in "pointing observing mode", characterized by long observations called Observation Blocks (OBs), typically of 2-4 weeks duration, following a predefined baseline Pointing Plan, mostly concentrated along the Galactic plane. On November 4, 2009, AGILE scientific operations were reconfigured following a malfunction of the rotation wheel occurred in mid October, 2009. The satellite is currently operating regularly in "spinning observing mode", surveying a large fraction (about 70\%) of the sky each day.

Since December 21, 2012 due to ASI's Malindi ground station technical problems, the acquisition of telemetry data from the AGILE satellite has been reduced. 


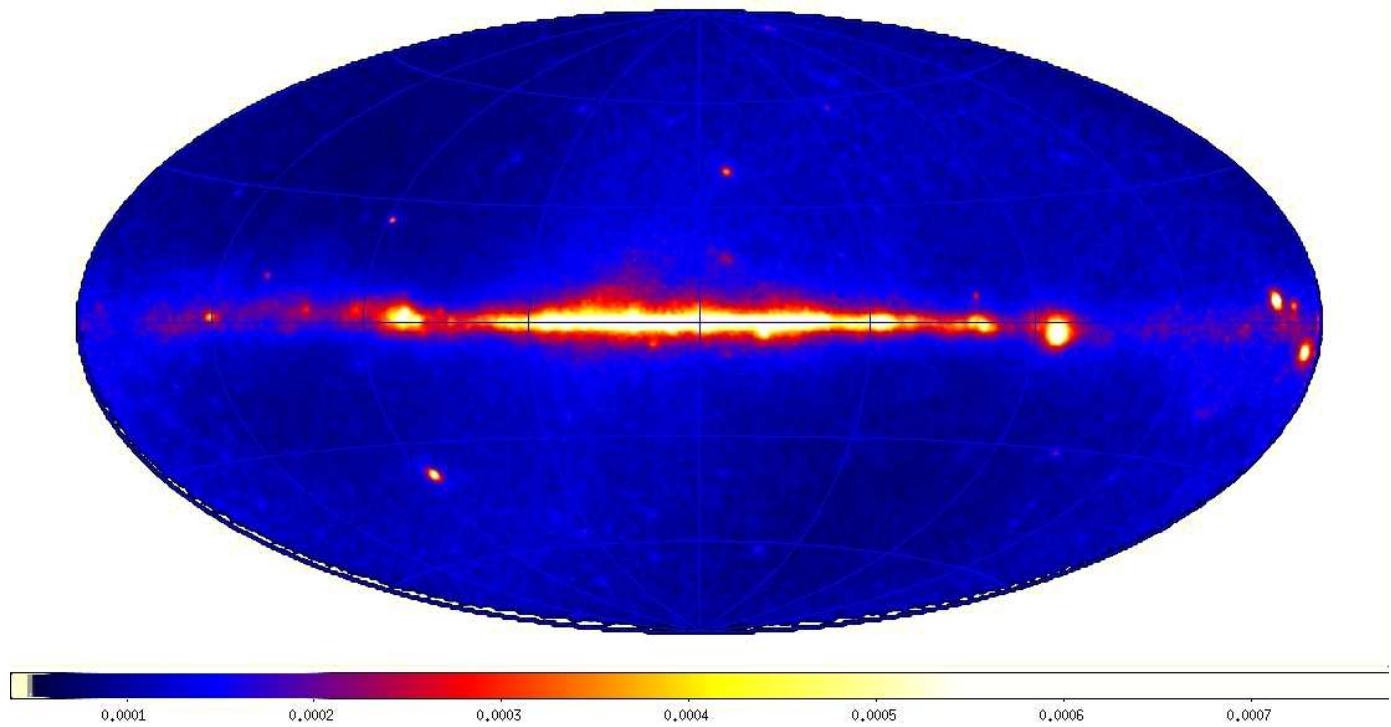

Figure 1: Whole sky AGILE intensity map $\left(\mathrm{ph} \mathrm{cm}^{-2} \mathrm{~s}^{-1} \mathrm{sr}^{-1}\right)$ in Galactic coordinates and Aitoff projection, for energies $E>100 \mathrm{MeV}$, accumulated during the first $\sim 6$ years of observations up to December, 2012 (pointing + spinning observing modes).

Malindi downlink efficiency for AGILE-GRID data is currently at a daily level of about $80 \%$. All AGILE payload functions are nominal, and the daily GRID skymonitoring activities are fully operational, despite the telemetry gaps in the archive due to intermittent data acquisition at the ground station.

The AGILE quicklook (QL) alert system is composed of two independent automated analysis parts: the QL Scientific pipeline running at ADC (Pittori et al., 2013) and the AGILE-GRID Science Alert System (SAS) pipeline running at the INAF-IASF Bologna (Bulgarelli et al., 2013). Data are automatically analyzed at every downlink and accumulated on different timescales. Lightcurves of potential gamma-ray sources are produced using blind search techniques and crosscorrelation with a reference list of known gamma-ray emitters. As proper flux thresholds are exceeded, alerts for transient gamma-ray sources are automatically generated and notified through e-mails, SMS messages, and also through a dedicated application for smartphones and tablets. These alerts are crosschecked, and a manual analysis is performed for the most interesting candidate gamma-ray sources.

The daily monitoring activity of the AGILE QL alerts resulted in the publication of 104 ATel and 40 GCN up to May, 2013, among which we mention the first detection of gamma-ray emission from microquasars above $100 \mathrm{MeV}$, from Cygnus X-3 and Cygnus $\mathrm{X}-1$ in the Cygnus region, the prompt alert of many gamma-ray flares from blazars and galactic transients, and the surprising discovery of gamma-ray flares from the Crab Nebula. The 2012 Bruno Rossi international Prize has been awarded to the PI, Marco Tavani, and the AGILE team for this important and unexpected discovery.

\section{New AGILE Catalogs and ASDC Interactive Web Tools}

The AGILE First Catalog of high-confidence $\gamma$-ray sources detected by AGILE during its first year of operations (Pittori et al., 2009) was recently updated using data covering the whole period of pointed observations, $\sim 2.3$ years, in Verrecchia et al. (2103) ("An updated list of AGILE bright gamma-ray sources and their variability in pointing mode"). Refined analysis of complex regions of the Galactic plane with improved event filter and calibrations yielded a new reference list, and a multi-source maximum likelihood analysis was performed over the timescale of the AGILE pointed Observation Blocks (OB) resulting in an updated list of 54 sources. Eight 1AGL sources, with significance above $4 \sigma$ from the analysis over the entire observing period, were not detectable on the $\mathrm{OB}$ timescale either due to low OB exposures and/or to their positions within complex galactic regions. The new AGILE-GRID variability catalog (Verrecchia et al., 2103) is also available from the ADC webpages as an interactive web ta$b^{1}{ }^{1}$. In the on-line version, light curves from fluxes in each OB are accessible within the ASDC Data Explorer,

\footnotetext{
${ }^{1} \mathrm{http}: / /$ www.asdc.asi.it/agile1rcat/
} 
in the AGILE-GRID data products tab. The ASDC Data Explorer is a multimission web tool which allows the user to browse both internal multimission archive catalogs (grouped by energy band) and external catalogs from other services (VIZIER, NED, SIMBAD etc.), providing an easy way to explore and cross-correlate large data sets from radio to $\mathrm{TeV}$. Moreover by using the ASDC SED builder tool (V3.0) it is possible to build and handle spectral energy distributions (SEDs), time resolved SEDs, and multi-frequency lightcurves. Virtual Observatory (VO) tools like TOPCAT can also be used to handle SED data in the time domain.

The AGILE reference list of bright gamma-ray sources above $100 \mathrm{MeV}$ over the first 2.3 years of observations thus includes at the moment 62 sources, as compared to the 47 1AGL in the First Catalog. Work is in progress to build new complete catalogs of AGILEGRID sources over the whole observing period (pointing+spinning).

The MCAL instrument of the AGILE satellite can observe the high-energy part of gamma-ray bursts (GRB) from $350 \mathrm{keV}$ to $100 \mathrm{MeV}$ with good timing capability, and the AGILE-MCAL GRB catalog, including 85 GRB observed from April 2007 to October 2009, was recently published in Galli et al. (2013). An online version of the new AGILE-MCAL GRB cata$\log$ is available from the ADC web pages ${ }^{2}$. We adapted the ASDC Data Explorer to provide the user with additional services dedicated to Gamma-ray Bursts through a GRB Explorer tool. The interactive web table in this case contains all the data for the published GRBs, including spectral data for 21 bursts, plus some supplementary information on other 7 events. Light Curves and Energy Spectra (when available) can be accessed from the GRB Explorer tool, in the AGILE MCAL Data Products tab. It is also possible to browse External Services to look for GRB-related data products from other missions, such as Swift-XRT light curve from Leicester repository, Swift-BAT product analysis, FermiGBM Quicklook lightcurve, GCNs and Blog for the chosen GRB.

\section{AGILE Science Highlights}

Fig. 1 shows the total gamma-ray intensity above 100 $\mathrm{MeV}$ as observed by AGILE up to December, 2012, during the first $\sim 6$ years of observations. We present here a selection of the main AGILE science highlights and some recent updates.

- AGILE has provided the first detection of a colliding wind binary (CWB) system above $100 \mathrm{MeV}$ in the $\eta$-Carinae region (Tavani et al., 2009b). AGILE detected a gamma-ray source (1AGL J1043-5931, now 1AGLR J1044-5944) consistent with the position of the

\footnotetext{
${ }^{2}$ http://www.asdc.asi.it/mcalgrbcat/
}

CWB massive system $\eta$-Car during the time period 2007 July - 2009 January. A 2-day gamma-ray flaring episode was also reported on 2008 Oct. 11-13, possibly related to a transient acceleration and radiation episode of the strongly variable shock in the system. A revised gamma-ray source list in the complex Carina region shown in Fig. 2 has been published in Verrecchia et al. (2013).

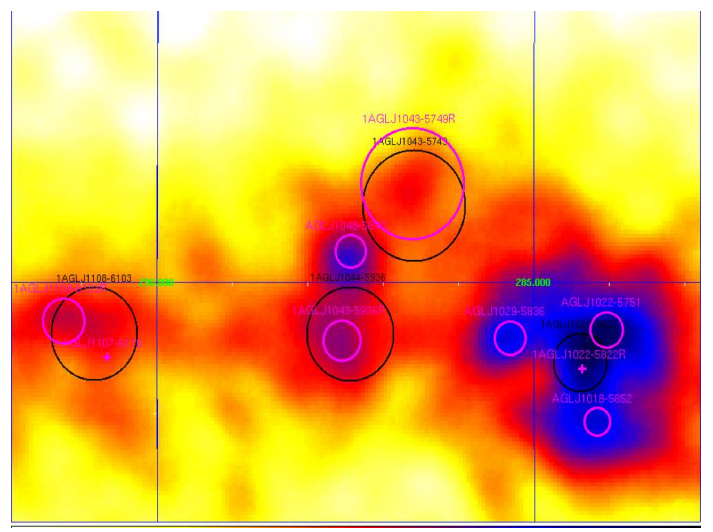

Figure 2: AGILE intensity map of the Carina region above $100 \mathrm{MeV}$ over $\sim 2.3$-year pointing mode observation period. New sources and revised $(\mathrm{R})$ positions for 1AGL sources are indicated in magenta, the 1AGL error circles are indicated in black.

- The AGILE detection of gamma-ray emission from the PWN Vela-X, described in a Science paper (Pellizzoni et al., 2010), has been the first experimental confirmation of gamma-ray emission (E>100 MeV) from a pulsar wind nebula. This result constrains the particle population responsible for the $\mathrm{GeV}$ emission and establishes a class of gamma-ray emitters that could account for a fraction of the unidentified galactic gamma-ray sources. Subsequently the NASA Fermi satellite has confirmed the Vela-X gamma-ray detection, and has also firmly identified other 4 pulsar wind nebulae plus a large number of candidates.

- Gamma-ray flaring activity for a source positionally consistent with Cygnus X-1 microquasar was reported twice by AGILE (Bulgarelli et al., 2010; Sabatini et al., 2010a and 2010b). AGILE extensive monitoring of Cygnus X-1 in the energy range $100 \mathrm{MeV}-3$ GeV during the period 2007 July - 2009 October confirmed the existence of a spectral cutoff between 1-100 $\mathrm{MeV}$ during the typical hard X-ray spectral state of the source. However, even in this state, Cygnus X-1 is capable of producing episodes of extreme particle acceleration on 1-day timescales, and even shorter lived flares in the $\mathrm{TeV}$ range as detected by MAGIC in 2006 (Albert et al., 2007). Gamma-ray flares of Cygnus X-1 detected 
by AGILE have been recently confirmed for the first time by a reanalisis of Fermi-LAT data (Bodaghee et al., 2012).

- AGILE detected for the first time several gammaray flares from Cygnus X-3 microquasar and also a weak persistent emission above $100 \mathrm{MeV}$ from the source (Tavani et al., 2009c). Gamma-ray flares occur either in coincidence with low hard-X-ray fluxes or during transitions from low to high hard-X-ray fluxes, and usually appear before major radio flares, following a clear repetitive pattern. This important AGILE finding has been confirmed by Fermi-LAT, which also detected the orbital period (4.8 hours) of the binary system in gammarays, securing unambigously the temporal signature of the microquasar (Abdo et al., 2009). In the 9 days from December 2 to December 11, 2009 AGILE and Fermi were able to answer a long-lasting question: Cygnus $\mathrm{X}$ 3 system is able to accelerate particles up to relativistic energies and to emit gamma-rays above $100 \mathrm{MeV}$.

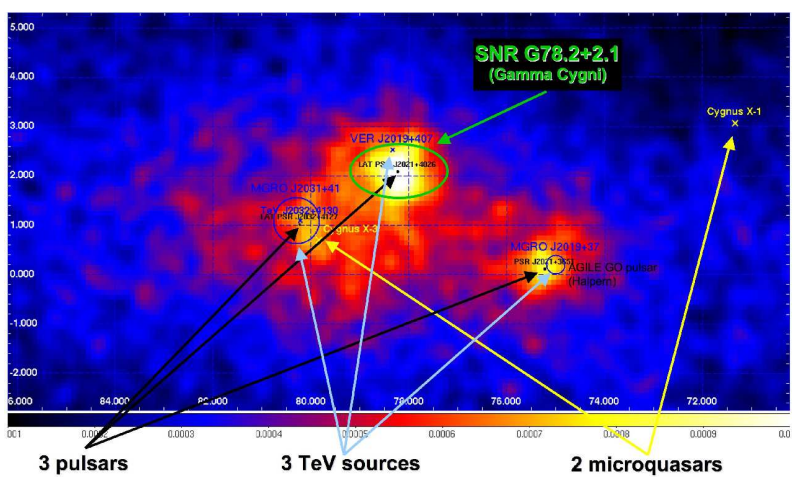

Figure 3: The Cygnus region in gamma-rays: AGILE intensity map from $100 \mathrm{MeV}$ to $10 \mathrm{GeV}$. Data taken in $\sim 2$-year pointing observing mode, from Nov. 2007 to Oct. 2009. Figure adapted from G. Piano presentation, 9th AGILE Workshop, 2012.

- Understanding the origin of cosmic rays is one of the most important issues of high-energy astrophysics, and galactic Supernova Remnants (SNR) are considered to be an ideal laboratory to study Cosmic-Ray acceleration. Experimental data analysis below $\mathrm{E}=200 \mathrm{MeV}$ is crucial to discriminate between theoretical models, since in this energy range hadronic and leptonic emission spectra have a well distinct behavior due to a steepening of the hadronic spectrum due to the neutral pion emission, which is missing in the leptonic case. The AGILE gamma-ray imager reaches its optimal sensitivity just at the energies in the $50 \mathrm{MeV}$ - a few $\mathrm{GeV}$ range at which neutral pions produced by proton-proton interactions radiate, and it was the first to discover a pattern of gamma-ray emission from the supernova remnant W44 that, combined with the observed multifrequency properties of the source, can be unambiguously attributed to

\footnotetext{
${ }^{3}$ http://www.asdc.asi.it/11thagilemeeting/program.php
}

accelerated protons interacting with nearby dense gas. This important AGILE result was reported in (Giuliani et al., 2011), and recently confirmed by new Fermi-LAT data (Ackermann et al., 2013) Before AGILE and Fermi observations a direct identification of sites in our Galaxy where proton acceleration takes place was elusive.

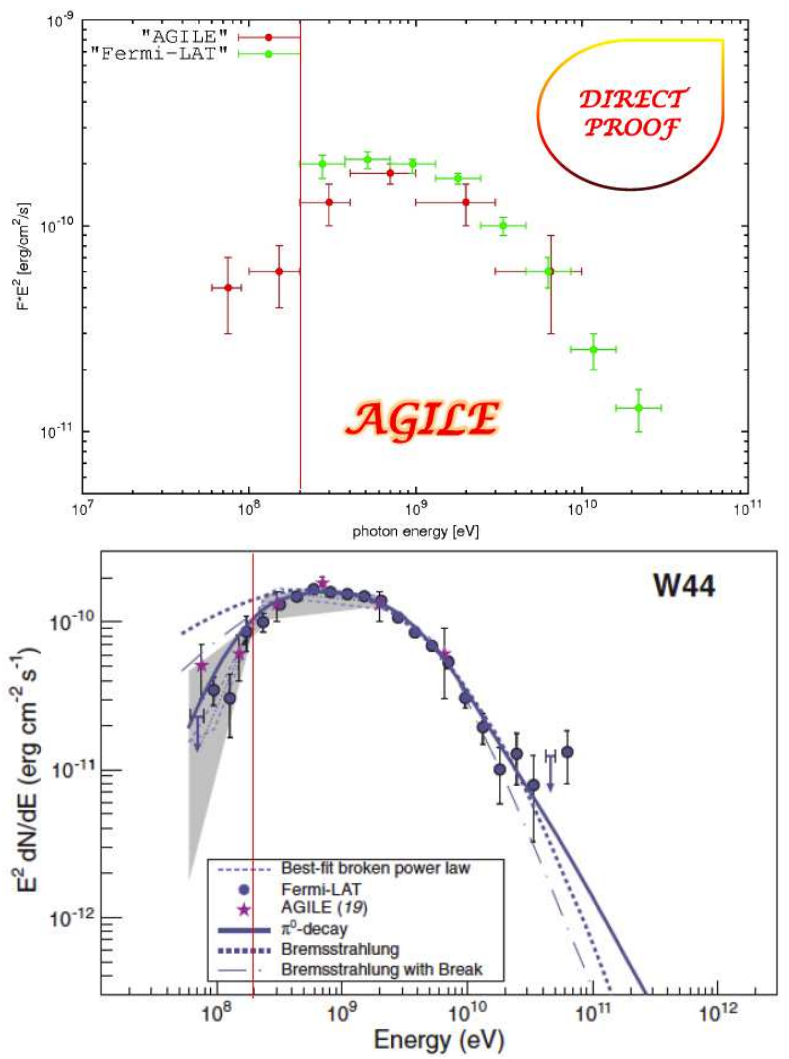

Figure 4: The W44 Supernova Remnant spectral energy distribution in gamma-rays. Upper panel: the first direct evidence of the pion bump from AGILE data below $200 \mathrm{MeV}$ (Giuliani et al., 2011). Lower panel: new Fermi-LAT data below $200 \mathrm{MeV}$ confirm the observed steepening due to the neutral pion emission (Ackermann et al., 2013).

Preliminary results from a new detailed study of the SNR Gamma-Cygni with AGILE have been presented by G. Piano during the $11^{\text {th }}$ AGILE Workshop ${ }^{3}$ (Piano et al., paper in progress). From AGILE data analysis there are hints of non-thermal emission possibly related to shock-cloud interactions in the North-Western part of the shell.

- The AGILE Minicalorimeter is also detecting Terrestrial Gamma-Ray Flashes (TGFs), intense and brief pulses of gamma-rays originating in the Earth atmosphere, and associated with thunderstorm activity. TGFs last a few thousandths of a second, and produce gamma-ray flashes up to $100 \mathrm{MeV}$, on timescales as low as $<5 \mathrm{~ms}$ (Marisaldi et al., 2010a). AGILE joins other satellites in detecting TGFs, but its unique capa- 
bility of detecting photons of the highest energies within the shortest timescales makes it an ideal instrument to study these impulsive phenomena. AGILE data have shown for the first time that TGF cumulative spectrum at high energy deviates from a power law with exponential cutoff model and can be better fit with a broken power law with significant counts above background up to $100 \mathrm{MeV}$. The crucial AGILE contribution to TGF science is thus the discovery that the TGF spectrum extends well above $40 \mathrm{MeV}$, and that the high energy tail of the TGF spectrum is harder than expected. Combined observations of high-energy photons detected by the AGILE-GRID detector have also provided the first direct localization of TGFs in gamma-rays, within a region clustered around the sub-satellite point (Fuschino et al., 2011). Thanks to its very low inclination orbit in the \pm 2.5 latitude band, AGILE provides the largest TGF surface density across the Equator available up to now. As statistics grows, AGILE data show that the TGF sample is possibly composed by two distinct populations: a low-energy population, consistent with typical TGF characteristics, and a high-energy population, with quite distinct features (Tavani et al., 2011; Marisaldi et al., 2012).

- Crab Nebula variability: The surprising discovery by AGILE of variable gamma-ray emission above $100 \mathrm{MeV}$ from the Crab Nebula in Sept. 2010 (Tavani et al., 2010; Tavani et al., 2011b), and the FermiLAT confirmation (Buehler et al., 2010; Abdo et al., 2011) started a new era of investigation of the Crab system. The 2012 Bruno Rossi international Prize has been awarded to the PI, Marco Tavani, and the AGILE team for this important and unexpected discovery.

Astronomers have long believed the Crab to be an almost ideal standard candle, a nearly constant source (at a level of few percent) from optical to gamma-ray energies (Meyer et al., 2010), with possible long-term nebular flux variations over a few-year timescale reported in the hard X-ray range. On September 2010 AGILE detected a rapid gamma-ray flare over a daily timescale, see Fig.4, and thanks to its rapid alert system, made the first public announcement on September 22, 2010. This finding was confirmed the next day by the Fermi Observatory. AGILE had also previoulsy detected a giant flare from the Crab in October, 2007 during the initial Science Verification Period of the satellite, and in the First AGILE Catalog paper (Pittori et al., 2009), it was reported that anomalous episodic flux values observed from the Crab in 2007 were under investigation. We know now of five major gamma-ray flares from the Crab Nebula detected by the AGILE-GRID and Fermi-LAT, up to May 2013. Gamma-ray data provide evidence for particle acceleration mechanisms in nebular shock regions more efficient than previously expected from current theoretical models. We estimate a recurrence rate for strong gamma-ray flares of $\sim(1-2)$ /year.

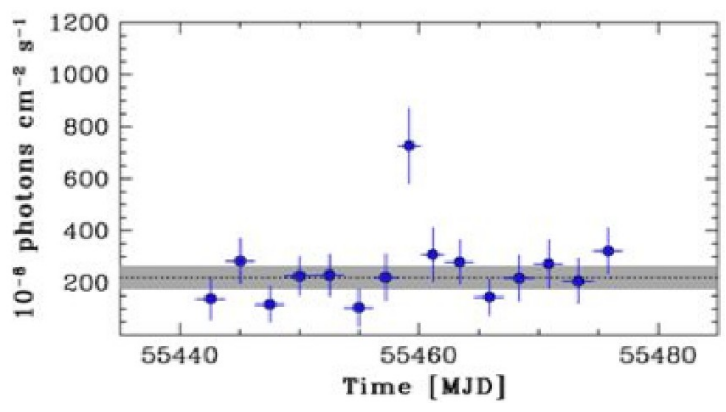

Figure 5: The Crab Nebula flare in September 2010, as observed by AGILE at energies above $100 \mathrm{MeV}$, (Tavani et al., 2011b).

A recent study (Striani et al., 2013 ) of the flux and spectral variability of the Crab Nebula above $100 \mathrm{MeV}$ on different timescales, ranging from days to weeks, both in AGILE-GRID and in Fermi-LAT data, also gives evidence for week-long and less intense episodes of enhanced gamma-ray emission that we call "waves" which can occur by themselves or in association with shorter flares. Both the flaring and "wave" events can be related to a population of accelerated electrons consistent with a mono-chromatic or relativistic Maxwellian distribution. Gamma-ray observations challenge standard Magneto-hydrodynamics models of nebular emission.

\section{Conclusions}

The study of cosmic gamma-rays in the energy range from a few tens of $\mathrm{MeV}$ to a few tens of $\mathrm{GeV}$ is only possible from space due to atmospheric absorption. With the successful launch of the new generation gamma-ray space ASI telescope AGILE on April 2007, followed by NASA Fermi on June 2008, gamma-ray astrophysics entered a new era. AGILE is substantially improving our knowledge on various known gamma-rays sources, such as supernova remnants and black hole binaries, pulsars and pulsar wind nebulae, blazars and Gamma Ray Bursts. Moreover, AGILE has contributed to the discovery and study of new galactic gamma-ray source classes, and of galactic gamma-ray transients. The Mission is also giving a crucial contribution to the study of the terrestrial gamma-ray flashes seen in the Earth atmosphere. Gamma-ray observations challenge standard theoretical models of particle acceleration and dynamics under extreme matter conditions.

\section{Acknowledgement}

Research partially supported through the ASI grants $\mathrm{I} / 089 / 06 / 2, \mathrm{I} / 042 / 10 / 0$ and I/028/12/0. 


\section{References}

[1] Abdo, A. A., et al. 2009, Science, 326, 1512. doi:10.1126/science.1182174

[2] Abdo, A. A., et al. 2011, Science, 331, 739. doi:10.1126/science.1199705

[3] Ackermann, M. et al., 2013, Science 339, 807.

[4] Albert J. et al, 2007, ApJ, 665, L51.

[5] Barbiellini, G. et al., 2001, AIP Conf. Proc. 587, 754.

[6] Bodaghee, A. et al., 2013, ApJ 775, 98B. doi:10.1088/0004-637X/775/2/98

[7] Buehler, R. et al., 2010, ATel \#2861.

[8] Bulgarelli, A. et al., 2010, ATel \#2512.

[9] Bulgarelli, A. et al., 2013, to appear in ApJ.

[10] Chen, A. et al., 2013, A\&A 558, A37.

[11] Galli, M., et al., 2013, A\&A 553.

[12] Giuliani, A. et al., 2011, ApJ 742, L30.

[13] Feroci, M. et al., 2007, NIM A 581, 728.

[14] Fuschino, F. et al., Geophys. Res. Lett. 38, L14806.

[15] Labanti, C. et al., 2006, SPIE, Volume 6266, 62663Q.

[16] Marisaldi, M., et al., 2010a, Journal of Geophys. Res. (Space Physics), 115, A00E13. doi:10.1029/2009JA014502

[17] Marisaldi, M., et al., 2010b, Phys. Rev. Lett. 105.

[18] Marisaldi, M., et al., 2012, EGU2013-4809, and paper in progress.

[19] Meyer, M. et al., 2010, A\&A, 523.

[20] Pellizzoni, A. et al., 2010, Science, Vol. 327 no. 5966, 663. doi:10.1126/science. 1183844
[21] Pittori, C., et al., 2009, A\&A 506, 1563-1574.

[22] Pittori, C., et al., 2013 Nucl. Phys. B. (Proc. Suppl.) 239-240, and paper in preparation.

[23] Prest, M. et al., 2003, NIM A, 501, 280.

[24] Sabatini, S. et al.,2010a, ATel \#2715.

[25] Sabatini, S. et al., 2010b, ApJ 712, L10. doi:10.1088/2041-8205/712/1/L10

[26] Striani, e. et al., 2013, ApJ 765, 52.

[27] Tavani, M. et al., 2009a, A\&A, 502, 1015.

[28] Tavani, M., et al., 2009b, ApJ 698, L142.

[29] Tavani, M. et al., 2009c, Nature 462, 620-623. doi:10.1038/nature08578

[30] Tavani, M. et al., 2010, ATel \#2855.

[31] Tavani, M. et al., 2011a, Phys. Rev. Lett. 106.

[32] Tavani, M. et al., 2011b, Science, 331, 73. doi:10.1126/science. 1200083

[33] Verrecchia, F., et al., 2013, A\&A 558, A137.

\section{DISCUSSION}

LUIGI PIRO: How can you distinguish between Inverse Compton and $\pi^{0}$ decay in the GeV range (question to Manami Sasaki talk on SNRs, Tue 28 afternoon).

CARLOTTA PITTORI Comment: My comment to Luigi Piro question is that, as I have shown in my talk, at least in some cases as W44, in which middle aged SNR interacts with Molecular Clouds, the new infrared Planck data are very much constraining, and the overall multiwaveleght picture seem to largely favour $\pi^{0}$ decay and hadronic models over the leptonic ones. 